\title{
Two separate nodules of Merkel cell carcinoma occurring concomitantly on one cheek
}

\author{
Woosuk Hwang ${ }^{1}$, \\ Mi Seon Kang ${ }^{2}$, \\ Jin Woo Kim ${ }^{1}$ \\ Departments of ${ }^{1}$ Plastic and \\ Reconstructive Surgery and ${ }^{2}$ Pathology, \\ Inje University School of Medicine, \\ Busan, Korea
}

\begin{abstract}
Merkel cell carcinoma (MCC) is a rare and highly aggressive neuroectodermal carcinoma arising from mechanoreceptor Merkel cells. Multiple MCCs are even rarer. We report a case of two independent MCCs simultaneously present in the cheek of a patient, which were effectively and esthetically treated using a cheek flap. Punch biopsy performed in a 60-year-old woman admitted with a chief complaint of two skin-colored hard nodules in her left cheek, accompanied by an itching sensation, was suggestive of MCC. Accordingly, we performed sentinel lymph node biopsy through the modified Blair incision under general anesthesia, in cooperation with the head and neck surgery department. The defect was covered with a cheek flap by slightly extending the existing incision following wide excision with a safety margin of $1 \mathrm{~cm}$. This paper is significant in that it introduces an effective reconstruction technique that maintains function using a cheek flap for the management of this rare case. In addition, this paper is the first to classify multiple MCCs according to the time of onset. We believe that this paper presents an effective alternative reconstruction technique with sentinel node biopsy through the modified Blair incision.
\end{abstract}

Keywords: Carcinoma, Merkel cell / Cheek / Surgical flaps

\section{INTRODUCTION}

Merkel cell carcinoma (MCC) is a rare and highly aggressive neuroectodermal carcinoma that arises from mechanoreceptor Merkel cells [1]. It was first characterized as a distinct clinical entity in 1972 and was reported to have an annual incidence of 0.6 per 100,000 population, which has been increasing by $8 \%$ yearly $[2,3]$. The major risk factors include polyomavirus infection, immunosuppression, and ultraviolet (UV) radiation exposure. In $81 \%$ of the cases, MCC is found in areas of UV-exposed skin. Among such cases, $36 \%$ are found in the head and neck region, with the cheeks being the most common location [4]. MCC is rare, and cases of multiple MCCs in the same person

Correspondence: Jin Woo Kim

Department of Plastic and Reconstructive Surgery, Busan Paik Hospital, Inje

University School of Medicine, 75 Bokji-ro, Busanjin-gu, Busan 47392, Korea

E-mail: jinooda@hanmail.net

Received October 5, 2018 / Revised November 3, 2018 / Accepted November 12, 2018 are even rarer $[5,6]$. We report the case of two independent but synchronous MCCs in the left cheek of a patient, which were effectively and esthetically treated using a cheek flap.

\section{CASE REPORT}

A 60-year-old woman with no underlying disease discovered two skin-colored hard, pruritic nodules, approximately $1 \mathrm{~cm}$ in size, in her left cheek 3 weeks before hospitalization. Punch biopsy performed following her visit to the department of dermatology revealed pathological findings of MCC, which led to consultation with the department of plastic surgery. No regional lymphadenopathy was observed. Enhanced computed tomography (CT) and positron emission tomography (PET) CT of the head, neck, and chest areas to detect regional or distant metastasis revealed no suspicious findings. The two nodules appeared similar on a photograph (Fig. 1A) and PET CT image. 

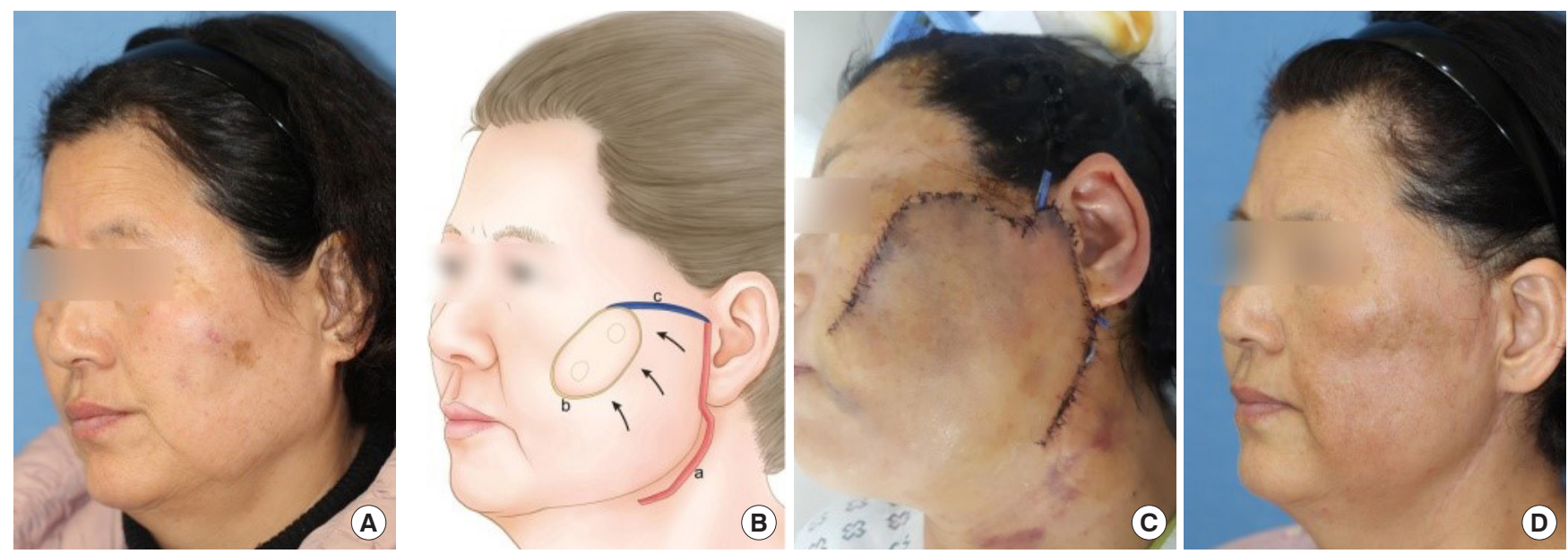

Fig. 1. (A) Preoperative photograph. (B) The $5 \times 2.5 \mathrm{~cm}$ sized cheek skin defect. After wide excision (b) was covered by elevating a lateral-based cheek flap through a small additional incision (c) added to the modified Blair incision (a). (C) Postoperative 1 day and (D) postoperative 7 months.
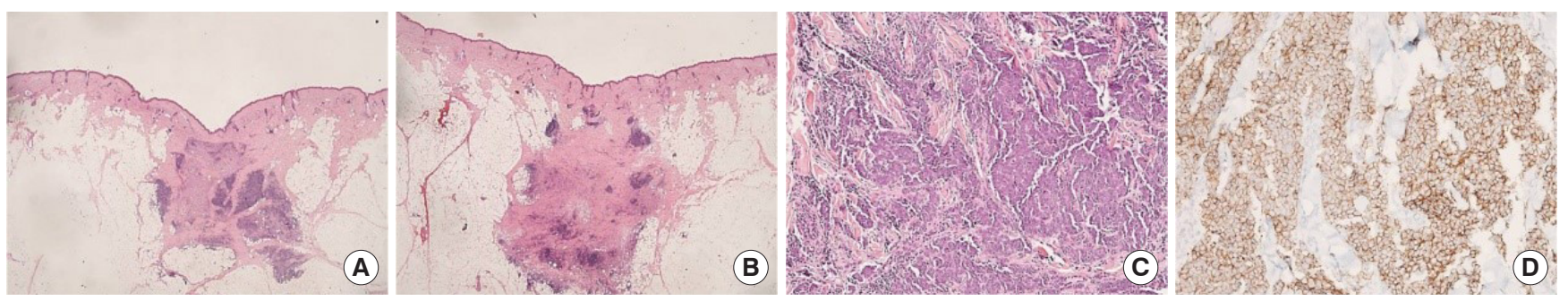

Fig. 2. Histologic findings of mass $1(\mathrm{~A})$ and mass $2(\mathrm{~B})(\mathrm{H} \& \mathrm{E}, \times 10)$. (C) Monotonous tumor cells are infiltrated in dermis $(\mathrm{H} \& \mathrm{E}, \times 100)$ and (D) they are CD56 positive (immunohistochemistry, $\times 100$ ).

The patient was admitted 1 day before surgery and underwent preoperative lymphoscintigraphy. Surgery was performed under general anesthesia in cooperation with the head and neck surgery department. First, a modified Blair incision for left partial parotidectomy and sentinel lymph node biopsy was made by the head and neck surgeon. The findings from frozen-section histological examination indicated no tumor. Subsequently, we performed wide excision with a 1-cm safety margin for the two nodular lesions. Frozen-section examination revealed MCCs in both nodules. All resected margins were clear. For the skin defect $\left(5 \times 2.5 \mathrm{~cm}^{2}\right.$ in size $)$, the modified Blair incision made by the head and neck surgeon was extended to cover the defect with a laterally based cheek flap elevation (Fig. 1B and C).

Postoperative permanent pathological findings indicated MCCs in both lesions (Fig. 2). The patient was discharged 10 days postoperatively, without complications, such as hematoma, seroma, and nerve injury, except slight skin necrosis distal to the flap. Two months postoperatively, adjuvant radiation therapy (total $56 \mathrm{~Gy}, 28$ fractions) was initiated. Scarring because of the therapy was observed at 7 months postoperatively, but it was nearly invisible (Fig. 1D). The patient is still undergoing regular follow-up, and no findings of local recurrence or metastasis were observed 3 years postoperatively.

\section{DISCUSSION}

MCC is a rare, aggressive cancer with a local recurrence rate of $35 \%$ to $40 \%$ and an overall 3-year survival rate of 50\% [7]. Except for multiple MCCs arising from local recurrence, multiple MCCs (cutaneous neuroendocrine carcinomas) in the same person can be divided into five types according to the time of onset: metachronous metastatic MCCs [6,8]; spontaneous multiple metastatic MCCs $[9,10]$; in-transit metastatic MCCs $[6,11]$; metachronous primary MCCs [6,12]; and spontaneous multiple primary MCCs [13].

Metachronous metastatic MCC is a primary MCC with distant metastasis. The prevalence of distant metastasis in MCC cases is approximately $6 \%$ to $7 \%$ [14]. Among such cases, the proportion of cases with the skin being the metastatic site is approximately $35 \%$ [15]. Primary and subsequent metastatic MCCs are found in $2 \%$ to $2.5 \%$ of all MCC cases.

Spontaneous multiple metastatic MCCs refer to multiple skin 
lesions due to cutaneous metastasis of primary neuroendocrine carcinoma occurring in the pancreatic and gastrointestinal tissues and are very rare, such that only a few cases have been reported so far $[9,10]$.

In-transit metastatic MCCs are uncommon, accounting for only $4 \%$ of all MCCs, and represent a regional condition that occurs in close spatial proximity to and independent of lymphatic metastases between the primary tumor site and a regional nodal basin. Although they may synchronously present with a solitary or primary lesion, they commonly appear as metachronous multiple nodules after primary tumor resection and mostly have poor outcomes [11].

Metachronous primary MCCs are "second primary" MCCs that develop after the onset of an existing primary MCC. They are differentiated from metastatic MCCs on the basis of genetic differences determined through genome analysis $[6,12]$.

Finally, spontaneous multiple primary MCCs refer to the simultaneous occurrence of different types of primary MCCs and are rarely reported. They must be differentiated from the in-transit metastatic MCCs or spontaneous multiple metastatic MCCs by genetic study or cancer screening [13].

In our case, the skin lesions occurred in close proximity to each other $(2-3 \mathrm{~cm})$, but both lesions were discovered simultaneously. Moreover, the patient showed a good prognosis with no local recurrence or regional lymph node or distant metastasis during the 3-year follow-up. Therefore, even if the lesions were synchronous, they might have a slight difference in the time of onset, and the likelihood of the lesions being in-transit metastases with poor prognosis was low. As visceral neuroendocrine carcinoma was not found, multiple distant metastasis could be dismissed as well. Accordingly, a diagnosis of two stage I primary MCCs occurring spontaneously was likely. However, one limitation of the report is that genetic study was not performed to obtain a more accurate diagnosis.

The optimal treatment for MCC is controversial. As the incidence of regional lymphatic basin metastases can reach up to $50 \%-70 \%$, some surgeons prefer prophylactic lymph node dissection with or without radiotherapy, which can reduce the regional failure rate to $20 \%$ but is associated with considerable morbidity. Consequently, the sentinel node technique, which can enable optimal regional control and reduce the number of unnecessary dissections, is being widely used today [16]. The sentinel node technique is performed at our hospital, in cooperation with the head and neck surgery department. For cases of skin cancer, the head and neck department prefer the modified Blair incision for dissection of the parotid and adjacent lymph nodes because of the advantages of a less prominent scar and lower likelihood of transection of the greater auricular nerve [17].

For cheek defects, reconstruction using local or regional flaps with tissue from adjacent units is preferable [18]. However, when a moderate-to-large defect is created because of a wide excision, as in cases involving MCC, reconstruction using a skin graft may also be performed. A local or regional flap can retain skin color and uniformity of texture better than a skin graft, and it has not been associated with minor or complete graft loss caused by postoperative radiotherapy and pain or scar in the graft donor site [19]. Accordingly, it may be viewed as an esthetic method with maintenance of function but has the disadvantage of leaving an additional scar from the additional incision. However, with the modified Blair incision for sentinel node biopsy, which starts from the preauricular crease and courses around the ear lobule into an upper neck crease, as in our case, additional incision for cheek rotation advancement flap may be minimal. Moderate defects, which are approximately $5 \times 2.5 \mathrm{~cm}^{2}$ in size, can be covered without extending the incision below the upper neck crease. For larger defects, successful reconstruction could be achieved by extending the incision down to the neck or chest, as in cases of skin cancer in old age [20].

In summary, we performed esthetic reconstruction with maintenance of function using a cheek flap in a rare case of two synchronous MCCs occurring in close proximity to each other. To the best our knowledge, this paper is the first to classify multiple MCCs according to the time of onset. We also believe that this paper presents an effective alternative reconstruction technique with sentinel node biopsy through a modified Blair incision.

\section{NOTES}

\section{Conflict of interest}

No potential conflict of interest relevant to this article was reported.

\section{Ethical approval}

The study performed in accordance with the principles of the Declaration of Helsinki. Written informed consent was obtained.

\section{Patient consent}

The patient provided written informed consent for the publication and the use of her images.

\section{ORCID}

Woosuk Hwang https://orcid.org/0000-0002-0304-4178 
Mi Seon Kang https://orcid.org/0000-0002-9784-2444

Jin Woo Kim https://orcid.org/0000-0002-7660-843X

\section{REFERENCES}

1. Amaral T, Leiter U, Garbe C. Merkel cell carcinoma: epidemiology, pathogenesis, diagnosis and therapy. Rev Endocr Metab Disord 2017;18:517-32.

2. Hodgson NC. Merkel cell carcinoma: changing incidence trends. J Surg Oncol 2005;89:1-4.

3. Albores-Saavedra J, Batich K, Chable-Montero F, Sagy N, Schwartz AM, Henson DE. Merkel cell carcinoma demographics, morphology, and survival based on 3870 cases: a population based study. J Cutan Pathol 2010;37:20-7.

4. Raju S, Vazirnia A, Totri C, Hata TR. Treatment of Merkel cell carcinoma of the head and neck: a systematic review. Dermatol Surg 2014;40:1273-83.

5. Thakur S, Chalioulias K, Hayes M, While A. Bilateral primary Merkel cell carcinoma of the upper lid misdiagnosed as Basal cell carcinoma. Orbit 2008;27:139-41.

6. Harms KL, Lazo de la Vega L, Hovelson DH, Rahrig S, Cani AK, Liu CJ, et al. Molecular profiling of multiple primary Merkel cell carcinoma to distinguish genetically distinct tumors from clonally related metastases. JAMA Dermatol 2017; 153:505-12.

7. Snow SN, Larson PO, Hardy S, Bentz M, Madjar D, Landeck A, et al. Merkel cell carcinoma of the skin and mucosa: report of 12 cutaneous cases with 2 cases arising from the nasal mucosa. Dermatol Surg 2001;27:165-70.

8. Bose A. Nine cases of Merkel cell tumour. J R Soc Med 1997; 90:439-42.

9. Schmidt U, Metz KA, Schrader M, Leder LD. Well-differentiated (oncocytoid) neuroendocrine carcinoma of the larynx with multiple skin metastases: a brief report. J Laryngol Otol 1994; 108:272-4.

10. Vidulich KA, Donley SE, Duvic M. Multinodular cutaneous spread in euroendocrine tumor of the breast: an unusual presentation. Am J Clin Dermatol 2007;8:379-83.

11. Allen PJ, Bowne WB, Jaques DP, Brennan MF, Busam K, Coit DG. Merkel cell carcinoma: prognosis and treatment of patients from a single institution. J Clin Oncol 2005;23:2300-9.

12. Nagy J, Feher LZ, Sonkodi I, Lesznyak J, Ivanyi B, Puskas LG. A second field metachronous Merkel cell carcinoma of the lip and the palatine tonsil confirmed by microarray-based comparative genomic hybridisation. Virchows Arch 2005;446:27886.

13. Satter EK, Derienzo DP. Synchronous onset of multiple cutaneous neuroendocrine (Merkel cell) carcinomas localized to the scalp. J Cutan Pathol 2008;35:685-91.

14. Lemos BD, Storer BE, Iyer JG, Phillips JL, Bichakjian CK, Fang LC, et al. Pathologic nodal evaluation improves prognostic accuracy in Merkel cell carcinoma: analysis of 5823 cases as the basis of the first consensus staging system. J Am Acad Dermatol 2010;63:751-61.

15. Medina-Franco H, Urist MM, Fiveash J, Heslin MJ, Bland KI, Beenken SW. Multimodality treatment of Merkel cell carcinoma: case series and literature review of 1024 cases. Ann Surg Oncol 2001;8:204-8.

16. Wasserberg N, Schachter J, Fenig E, Feinmesser M, Gutman H. Applicability of the sentinel node technique to Merkel cell carcinoma. Dermatol Surg 2000;26:138-41.

17. Wormald R, Donnelly M, Timon C. 'Minor' morbidity after parotid surgery via the modified Blair incision. J Plast Reconstr Aesthet Surg 2009;62:1008-11.

18. Menick FJ. Reconstruction of the cheek. Plast Reconstr Surg 2001;108:496-505.

19. Bui DT, Chunilal A, Mehrara BJ, Disa JJ, Alektiar KM, Cordeiro PG. Outcome of split-thickness skin grafts after external beam radiotherapy. Ann Plast Surg 2004;52:551-6.

20. Al Shetawi AH, Quimby A, Fernandes R. The cervicofacial flap in cheek reconstruction: a guide for flap design. J Oral Maxillofac Surg 2017;75:2708.e1-2708.e6. 